\title{
Le poids des émotions en médecine périnatale...
}

\section{Quels fondements pour une démarche scientifique ?}

\section{The significance of emotional responses in perinatal medicine...} What is the basis for a scientific approach?

\author{
F. Molénat · J.-P. Visier \\ (C) Springer-Verlag France 2010
}

\begin{abstract}
"Perdre un enfant, c'est terrible, mais on peut l'assumer. Se sentir volé de ses émotions, de cela on ne se remet pas » (témoignage de parents à l'occasion d'une étude clinique sur les grossesses après mort périnatale, 1981)
\end{abstract}

Ces trois décennies ont vu des progrès considérables. Un voile se déchirait. Appuyés sur une meilleure sécurité technique et sur une bonne maîtrise de la mortalité périnatale, les médecins (re)découvraient une autre place, lorsque la vie malgré tout leur échappait : être là. S'il leur paraissait logique que des couples endeuillés changent d'équipe pour une nouvelle grossesse : ils virent au contraire revenir vers eux des parents confiants, qui s'étaient sentis reconnus et accompagnés dans l'épreuve. À la douleur, et son cortège de culpabilité, s'adjoignait un ressenti positif : la valeur des sentiments éprouvés dans la rencontre avec les professionnels.

Les parents nous ont beaucoup aidés. Dans les années 1980 leur témoignage produisit un effet de choc : « nous avons grandi, nous ne sommes plus les mêmes... quand nous passons devant l'hôpital où s'est éteint notre enfant, nous avons des images de vie et non de mort »... Pas d'étude randomisée, mais la conviction de quelques praticiens allait rapidement transformer les pratiques, comme une traînée de poudre. À la même époque, le concept de « lien mèreenfant » (qui intègrerait progressivement le père) et les dégâts ultérieurs s'ils n'étaient préservés dès la naissance, bouleversaient maternités et services de pédiatrie néonatale. Les premiers travaux sur l'attachement rencontraient une vérité humaine tapie en chacun des soignants.

Dans le même temps, arrivèrent les techniques de diagnostic anténatal, qui allaient accélérer la rencontre des disci-

F. Molénat $(\square)$

15, rue Cité-Bousquet, F-34090 Montpellier, France

e-mail : fmolenat@yahoo.fr

J.-P. Visier

Service MPEA, F-34295 Montpellier cedex 05, France plines et poser de nouvelles questions : comment annoncer, comment réanimer l'enfant dans la tête des parents, comment décider? On a largement décrit les grandes leçons de cette nouvelle approche : ne pas aller trop vite, se soucier du couple, parler d'un enfant et pas seulement d'un fotus, ne pas endormir lors de gestes décisifs... Être là, mais comment? S'il fallait retenir les plus fécondes de ces leçons, ce serait peut-être : tenir fermement le cadre médical pour permettre aux émotions des parents d'évoluer et non l'inverse. Résister aux demandes angoissées sans abandonner. Mais aussi : sortir de son isolement, tenir compte de l'avis d'acteurs à une autre place. Et là, nous assistâmes aux effets surprenants d'une approche coordonnée sur le psychisme parental - en particulier chez ceux que la vie avait rendus particulièrement vulnérables. L'accompagnement du diagnostic anténatal dans toutes ses étapes deviendrait un modèle pour bien d'autres situations complexes : la cohérence des professionnels entre eux, dans la juste distance avec les couples, permet à ces derniers de se reconstruire, malgré l'épreuve ou à travers elle. Nos armes classiques de psychothérapeutes pâlissaient devant la rapidité évolutive. En revanche, les effets structurants d'un soutien collectif permettraient d'affiner les modalités d'intervention psychologique et d'en accentuer la pertinence.

Ainsi, s'est élaborée une séméiologie de l'accompagnement qu'on nomme désormais " en réseau », et qui permet à toute une équipe de s'ajuster, avec ses propres critères somatiques, à l'évolution émotionnelle de chaque couple. L'analyse des séquelles psychologiques - et parfois somatiques - validait l'hypothèse : quelle que soit la vulnérabilité antérieure de la mère ou du père, on retrouvait dans les multiples détails de la prise en charge le grain de sable qui avait bloqué la fluidité psychique et figé l'impact traumatique. La métaphore de « chirurgie relationnelle » a couru. La rigueur offerte dans le présent pouvait ainsi déjouer les expériences passées qui font le lit du traumatisme. En revanche, un manque de clarté dans les places ou les avis, une attente injustifiée, risquaient de renforcer le sentiment 
de dévalorisation ou d'abandon déjà vécu : nous le retrouverions plus tard dans les consultations pour dépression maternelle, conflit conjugal, grossesse trop rapide et/ou tourmentée. Tenir ensemble l'objectivité des examens médicaux et la subjectivité de chaque couple soumis à des émotions violentes pour mieux cerner la «vérité »d'une situation devenait possible et enrichissait l'approche médicale : cette «manière de penser et d'être » allait servir de modèle dans bien d'autres problématiques, donnant toute sa plénitude à une «médicalisation raisonnée » du suivi de grossesse.

Et la science dans tout cela ? Les changements vécus au fil des années sous les coups de boutoir du bon sens et de l'engagement soignant posent la question de la connaissance. Comment se forge-t-elle, entre evidence-based medicine et intuition humaine ? La part nécessaire d'intersubjectivité entrave-t-elle la vérité des chiffres ? La conviction acquise après changement des attitudes - qui songerait à mener une étude comparative sur les pratiques en cours autour du décès périnatal — repose-t-elle sur des bases scientifiques qui protégeraient d'un trop de subjectivité ? La sécurité apportée aux équipes par une meilleure écoute des parents valide-t-elle les efforts entrepris ? Les soins personnalisés au bébé en pédiatrie relèvent-ils du respect ou d'une démonstration scientifique ? Les deux, dirons-nous. Toutes les voix (ou presque) — des neurophysiologistes et des généticiens aux psychologues se rejoignent: opposer l'inné et l'acquis, le biologique et l'environnemental, est désormais sans fondement.

P. Delion, dans un numéro précédent de cette revue, rappelait que Freud lui-même annonçait un écroulement possible de « tout l'édifice artificiel de nos hypothèses... de conceptions provisoires en psychologie ", nous préparant à " recevoir de la biologie les lumières les plus surprenantes ». Les attaques actuelles ne viennent-elles pas en partie de notre lenteur à ajuster ces hypothèses fécondes à l'évolution de la société et des connaissances ? Le difficile est peut-être d'accepter que, dans le registre de la vie psychique, l'émotion pèse plus lourd que les faits. Le contenu des informations livrées par une femme enceinte n'a de portée qu'au travers du halo d'affects et de représentations qui l'accompagnent, ce halo étant différent selon le contexte de la rencontre avec tel ou tel soignant. Comme le dit avec force dans son dernier ouvrage le neurologue L. Naccache : « nous percevons et nous interprétons en même temps ». Le réel n'est perçu qu'au travers de l'épaisseur de notre réalité psychique, que l'on soit parent, nourrisson, professionnel. Un gynécologue-obstétricien d'exercice libéral en témoigne : «Avant je les écoutais, mais je ne les entendais pas... parce que je n'avais pas les réponses. Parfois le malaise montait. Elles étaient stressées et me stressaient. Maintenant, je ne travaille plus seul. Je reste concerné, mais je sais que d'autres organisent les réponses. Maintenant, je les entends, sans y passer plus de temps ».

Reste une question en chantier : de qui relève la prise en compte des émotions ? On serait tenté d'en laisser la charge aux spécialistes du psychisme. Ce serait oublier que l'émotion s'enracine dans les traces corporelles liées à d'autres temps vécus, qu'elle est mobilisée tout autant dans le registre du soin somatique que celui de la verbalisation. L'adresse trop rapide à un psychothérapeute sur facteurs objectifs de risque court à l'échec. Le chemin d'une première alliance est parfois long pour que des ressentis enfouis remontent à la conscience, faute de quoi l'enfant viendra activement les explorer à travers ses symptômes. Bien plus, c'est l'éprouvé d'une sensation de protection dans le présent qui réactive ce qui a pu manquer dans une trajectoire chaotique : " J'appelle et on vient... », confie une femme hospitalisée en fin de grossesse pour angoisse à expression somatique: «Je n'avais jamais éprouvé cela ». Médecins, sages-femmes, puéricultrices, aides-soignantes, dans la proximité liée aux soins, se retrouvent impliqués dans le registre affectif, qu'ils le veuillent ou non. Ne pas s'y intéresser par prudence porterait en soi un risque d'impact sur l'autre — en négatif : ne pas se sentir reconnu dans sa propre singularité. Il est bien connu désormais que les conseils éducatifs tombent à plat si les parents n'ont pu dire leurs questions et inquiétudes. S'y intéresser, comment, jusqu'où, dans quel contexte de collaboration, ainsi s'est ouvert le vaste chantier qui n'est pas sans analogie avec le souci écologique : améliorer l'environnement fœtal, puis celui du nourrisson, tenir compte des ressources autant que des limites, ajuster finement l'intervention technique à ce qui fait sens pour les parents, etc. Tout cela relève-t-il de l'éthique, de la psychologie, d'une démarche « scientifique »? Pour les professionnels de la grossesse, la tâche n'est pas simple : le souci premier est de donner toutes ses chances à l'enfant. La complexité du développement humain ne fait pas partie de la formation de base, et les occasions restent rares d'évaluer après coup l'effet des attitudes périnatales.

Parallèlement aux vastes études longitudinales telles qu'Épipage, les rencontres organisées par les réseaux périnatals, les reprises de dossiers dans la continuité grossesse/petite enfance, la réflexion approfondie menée par néonatalogistes et obstétriciens sous la bannière de l'éthique en grande prématurité permettent de croiser les regards pour une approche globale. Une nouvelle culture se développe. Le dernier Plan périnatalité a ouvert de sérieuses possibilités : un cadre cohérent se dégage pour un suivi personnalisé de haute technicité dans les situations qui accumulent les facteurs de vulnérabilité, de l'anté- au postnatal, jusque dans les premiers mois de la vie. Ce chantier-là est bien entamé. Formations interdisciplinaires, dispositifs originaux de collaboration, guides de bonnes pratiques devraient baliser dans les années à venir ce nouveau chapitre de la médecine périnatale. 